\title{
Influencia del estudio personal en la adquisición de conocimientos
}

\author{
"The Influence of personal study on the aquisition of knowledge"
}

Josep Nebot-Cegarra ${ }^{a}$, Mercedes Campillob y Jorge Pérez

a Unitat d'Anatomia i d'Embriologia, Departament de Ciències Morfològiques, Facultat de Medicina, Universitat Autónoma de Barcelona (UAB).

${ }^{\mathrm{b}}$ Laboratori de Medicina Computacional, Unitat de Bioestadística, Facultat de Medicina, UAB.

${ }^{\mathrm{c}}$ Facultat de Ciències de la Salut i de la Vida. Universitat Pompeu Fabra.

Introducción. La actividad del docente suele ser la primera influencia formativa que recibe el alumno en el proceso de aprendizaje de un determinado tema, pero se consolida mediante el estudio personal. En el presente trabajo se ha determinado el grado de mejora obtenido tras el estudio personal en la puntuación alcanzada en una serie de cuestiones, que fueron previamente evaluadas justo después de ser enseñadas'.

Material y Métodos. Se efectuó una prueba a 66 alumnos inmediatamente después de realizar una actividad teoricopráctica de la asignatura Biología del Desarrollo y Teratogenia, que la repitieron días después. Se valoró la existencia de diferencias significativas en el grado de mejoría en función de las características de las cuestiones planteadas y del tiempo transcurrido entre ambas pruebas. Resultados. Hubo mejora en todos los tipos de cuestiones siendo significativa para las cuestiones en que los alumnos precisaron durante la sesión docente una actitud más activa que aquellas otras en que solamente debían observar. De estas últimas mejoraron más las de percepción auditiva. En las respuestas que requerían nombrar, la mejora significativa fue inversamente proporcional a la complejidad de los términos, pero los mejores resultados se obtuvieron en los términos con dificultad fonética alta. Los días transcurridos entre las pruebas no influyeron significativamente en los incrementos de la nota global.

Conclusiones. El estudio personal permite una mejora general en la comprensión de los contenidos, independientemente de su grado de dificultad en el aprendizaje a corto plazo.

Palabras clave: Educación médica. Evaluación. Aprendizaje. Comunicación no verbal. Embriología. Docencia universitaria.

Correspondencia:

Dr. Josep Nebot-Cegarra.

${ }^{a}$ Unitat d'Anatomia i d'Embriologia, Departament de Ciències

Morfològiques, Facultat de Medicina, Universitat Autónoma

de Barcelona.

08193 Bellaterra (Barcelona).

E-mail: Josep.Nebot@uab.es
Introduction: Personal study is fundamental to the consolidation of knowledge and learning. In our study we have established the degree of improvement achieved after personal study by comparing it with that obtained immediately after a learning experience'.

Material and Methods: 66 students on the course the "Developmental Biology and Teratogeny" took part in the study. They did a test immediately after a theorypractical activity, and this same test was then repeated as part of the final evaluation of the course. We observed the degree of learning improvement achieved in both of these tests based on the nature of the questions and the time that had passed between the two tests.

Results: The results were always better in the second test. It was superior in the learning experience in which the students were more actively involved. When they had only to observe, the questions taught without audiovisual support improved more than other ones where this methodology was used. The increase in learning was particularly apparent in questions that they had to answer with words less complex and with high difficult phonetically. The time lapse between the tests had no significant impact on the level of improvement.

Conclusions: Personal study allows for a general improvement in learning regardless of the degree of difficulty which is perceived in immediate learning.

Key words: Medical education. Evaluation. Learning. Nonverbal Communication. Embryology. Higher education.

\section{INTRODUCCIÓN}

La actividad del docente suele ser la primera influencia formativa que recibe el alumno en el proceso de aprendizaje de un determinado tema ${ }^{1}$. Su efectividad en la consolidación posterior de los conocimientos ha sido tanto realzada ${ }^{2}$ como cuestiona$\mathrm{da}^{3}$. De todos modos es el primer eslabón que debe 
engarzarse con muchos otros, tales como el estudio personal reiterado, la pausada reflexión, la ampliación de los contenidos, y la resolución de las dudas con el profesor.

Nuestro trabajo pretende determinar el papel del estudio personal en el grado de mejora del aprendizaje valorando la diferencia de puntuación obtenida al responder idénticas cuestiones en dos pruebas: la primera inmediatamente después de efectuar la actividad docente en que se aprendieron los temas, y la segunda tras un período de tiempo que permitió su estudio demorado. Para analizar el grado de mejora se han agrupado las cuestiones según el método utilizado en su enseñanza y según el tipo de pregunta. Se ha valorado también la posible influencia del tiempo transcurrido entre ambas pruebas.

\section{MATERIAL Y MÉTODOS}

\section{Alumnos: Número y características}

Participaron en el estudio 66 alumnos pertenecientes al segundo curso de la Licenciatura en Medicina de la Universitat Autònoma de Barcelona, matriculados en la asignatura optativa Biología del desarrollo y Teratogenia.

\section{Evaluaciones}

En dos días distintos los mismos alumnos se sometieron a dos pruebas que contenían tres preguntas coincidentes, en las que se planteaban un total de 10 cuestiones. Ambas pruebas valoraron el contenido de una actividad teoricopráctica de la antes citada asignatura, que no había sido enseñado previamente.

La primera evaluación se efectuó al final de cada sesión, sin previo aviso, con carácter voluntario y sin repercusión académica. El objetivo fue evaluar la influencia de la metodología en la adquisición rápida de conocimientos ${ }^{1}$. La segunda prueba, realizada con posterioridad, correspondía a la evaluación oficial de la actividad teoricopráctica y por tanto su fecha era conocida desde el principio del semestre. $\mathrm{Su}$ valor máximo en la nota final de la asignatura representaba 0'4 puntos sobre 10 .

Se omitió en todo momento la información acerca de la repetición parcial del temario y no existían precedentes de esta experiencia en cursos anteriores. Aparte de dos preguntas nuevas en la segunda prueba (no valoradas en este estudio), los factores diferenciales entre ambas pruebas fueron el tiempo transcurrido desde la realización de la sesión docente y la posibilidad de estudiar personalmente los temas objeto de evaluación.

\section{Procedimientos}

La organización, metodología docente y tipología de las cuestiones están explicadas en Nebot et al $^{1}$.

1. Valoración de los resultados. Cada una de las tres preguntas coincidentes incluía cuestiones que fueron valoradas independientemente, con 1 punto si era correcta o con 0 puntos en caso de error. La puntuación por tipo de cuestión consistió en la suma de los valores obtenidos en las cuestiones correspondientes a las diferentes tipologías y su posterior transformación a una escala de 0 a 10 puntos. A partir de la nota de cada prueba, obtenida sumando la puntuación de cada una de las diez cuestiones que comprendían las tres preguntas, se determinó la variable diferencia entre las dos pruebas (puntuación de la prueba final - puntuación de la prueba inicial). Se determinó asimismo la diferencia de puntuación que en cada cuestión obtuvo cada alumno. Se analizó la existencia de diferencias estadísticamente significativas entre los diferentes tipos de cuestión en cuanto a la diferencia de las puntuaciones entre las dos pruebas, así como en relación con la diferencia de la nota global para los distintos grupos de alumnos. Los alumnos se agruparon de acuerdo con los días transcurridos entre ambas pruebas (entre 12 y 20 días).

2. Análisis estadístico. Las hipótesis de normalidad se contrastaron con el test de KolmogorovSmirnov con la aproximación de Lilliefors. Cuando la comparación de la variación en las puntuaciones obtenidas según el tipo de cuestión se refería a dos grupos, se utilizó el test de la t de Student de datos apareados bajo el supuesto de normalidad o la prueba de los rangos con signo de Willcoxon en caso contrario; la comparación de más de dos grupos se realizó con un análisis de la varianza de medidas repetidas o la prueba de Friedman para valores normales o no normales, respectivamente. Los contrastes por grupo de alumnos en función del número de días transcurridos entre ambas pruebas se efectuaron con un análisis de la varianza de un factor. Todos los análisis fueron realizados con el programa estadístico SPSS 10.0 [SPSS Inc. 2000471 /id\}. El nivel de significación del estudio fue del $5 \%$.

\section{RESULTADOS}

En todas las cuestiones se obtuvo una mejora con el estudio personal. A continuación se detalla su grado de significación estadística.

\section{Según la recepción de la información}

Los datos estadísticos se muestran en la Tabla 1. 1. Según la percepción. No se obtuvieron diferen- 
Tabla 1. Resumen de los resultados estadísticos según la tipología de las cuestiones ${ }^{a}$

\begin{tabular}{|c|c|c|c|c|c|}
\hline & Mediab $^{b}$ & $\begin{array}{r}\text { Desviación } \\
\text { Típica }\end{array}$ & $\begin{array}{r}\text { Percentiles } \\
25 \\
\end{array}$ & 50 & 75 \\
\hline \multicolumn{6}{|l|}{ Tipo de percepción } \\
\hline Mixta & 3.9 & 2.6 & 2.5 & 5.0 & 5.0 \\
\hline Auditiva & 4.0 & 2.7 & 2.0 & 4.0 & 6.0 n.s. \\
\hline \multicolumn{6}{|c|}{ Actitud mínima requerida } \\
\hline Observación & 4.8 & 3.6 & 0.0 & 5.0 & 6.3 \\
\hline Acción personal & 3.4 & 3.8 & 0.0 & 3.3 & $6.7^{*}$ \\
\hline \multicolumn{6}{|l|}{ Actitud mínima requerida } \\
\hline Acción personal & 4.2 & 2.4 & 3.3 & 5.0 & 6.7 \\
\hline Observación & 2.8 & 3.2 & 0.0 & 2.5 & 5.0 \\
\hline Percepción mixta & 3.9 & 2.6 & 2.5 & 5.0 & 5.0 \\
\hline Percepción auditiva & 4.8 & 3.6 & 0.0 & 5.0 & $6.3 *$ \\
\hline \multicolumn{6}{|c|}{ Acción solicitada en la respuesta } \\
\hline Definir & 3.4 & 3.8 & 0.0 & 3.3 & 6.7 \\
\hline Nombrar & 4.2 & 2.4 & 3.3 & 5.0 & 6.7 n.s. \\
\hline \multicolumn{6}{|l|}{ Complejidad del término } \\
\hline Simple & 5.4 & 2.9 & 2.5 & 5.0 & 7.5 \\
\hline Complejo & 1.7 & 3.6 & 0.0 & 0.0 & 5.0 ** \\
\hline \multicolumn{6}{|l|}{ Dificultad fonética ${ }^{c}$} \\
\hline Baja & 3.6 & 3.6 & 0.0 & 5.0 & 5.0 \\
\hline Media & 1.9 & 3.5 & 0.0 & 2.5 & $5.0 *$ \\
\hline Alta & 7.0 & 3.4 & 5.0 & 10.0 & $10.0 * *$ \\
\hline
\end{tabular}

${ }^{a}$ No se considera la cuestión 10; ${ }^{b}$ Diferencias de puntuación entre ambas pruebas en escala de 0-10; ' La significación estadística de cada categoría se refiere a las categorías anteriores. ${ }^{*} p<0.05 ;{ }^{* *} p<0.001$. n.s., no diferencias significativas

cias estadísticamente significativas en la variación de las puntuaciones obtenidas en cada prueba en las cuestiones de percepción auditiva (explicadas sin iconografía) y las de percepción mixta (explicadas con iconografía)

2. Según la actitud mínima requerida al alumno. Se obtuvieron diferencias de puntuaciones significativamente mayores en las preguntas en que el alumno precisó tomar anotaciones o establecer secuencias del desarrollo (actitud mínima requerida de acción personal) que en aquellas en que al alumno se les entregó un texto escrito y por tanto podía estar exclusivamente observando al profesor y/o a la iconografía utilizada (actitud mínima requerida de observación)

3. Según la percepción y la actitud mínima requerida. Cuando se consideraron sólo las cuestiones que el alumno percibió auditivamente, se observó que aquellas en que requería una actitud mínima de observación tuvieron un aumento en las puntuaciones estadísticamente significativo respecto aquellas otras en las que el alumno precisó adoptar una actitud mínima de acción personal. Cuando se comparó la diferencia de las puntuaciones sobre las cuestiones que requirieron una actitud mínima de observación se apreció una mejoría significativamente mayor en aquellas que el alumno percibió auditivamente que en las percibidas también visualmente, mediante imágenes o preparaciones anatómicas (percepción mixta)

\section{Según el tipo de respuesta solicitada}

Los datos estadísticos se muestran en la Tabla 1.

1. Según la acción a realizar. No se obtuvieron diferencias significativas entre los cambios de puntuación obtenidos en las cuestiones según el tipo de acción a efectuar al responderlas: identificar (nombrar) los tipos de teratópagos representados en imágenes, o definir las anomalías placentarias indicadas.

2. Según el tipo de término a nombrar. En las cuestiones cuyas respuestas requirieron utilizar términos con un prefijo para identificar teratópagos 
(términos simples) la diferencia de las puntuaciones fueron significativamente mayores que las obtenidas en las cuestiones resueltas con términos con dos prefijos (términos complejos)

3._Según la dificultad fonética del término a nombrar. En las cuestiones cuyas respuestas correctas requerían utilizar términos con prefijos de raíces desconocidas previamente por el alumno (términos con dificultad fonética alta), el aumento de las puntuaciones fue significativamente mayor que en aquellas cuya respuesta tenía una dificultad fonética menor. Entre las cuestiones que se respondían con palabras cuyos prefijos eran de raíces conocidas, mejoraron significativamente más aquellas en que era más fácil establecer la relación del término con la imagen a identificar (términos con dificultad fonética baja) que en aquellas en que era más difícil establecer dicha asociación de ideas (términos con dificultad fonética media). El sufijo de todos los términos a nombrar coincidía.

\section{Según el tiempo transcurrido entre ambas pruebas}

Se muestran los datos estadísticos en la Tabla 2.

No se observaron diferencias estadísticamente significativas entre los cambios de nota global de los grupos de alumnos con diferentes días de separación entre ambas pruebas.

\section{DISCUSIÓN}

En el presente estudio hemos analizado cómo el estudio personal puede influir en la mejora significativa del aprendizaje. Con este fin se han calculado las diferencias de las notas obtenidas en cada una de las diez cuestiones que se plantearon a los alumnos, tanto en una prueba de comprensión que, por sorpresa, se realizó justo después de acabar una práctica, con contenido también teórico, como en la evaluación con contingencia académica de la actividad docente, que se hizo tiempo después, en una fecha previamente anunciada. Para no desvirtuar nuestro objetivo, se eligieron en la prueba temas que eran nuevos para los alumnos, ya que únicamente se les enseñó durante la actividad docente objeto de evaluación. Los alumnos acudían con algunos preconceptos enseñados en otros temas, pero que no permitían contestar la evaluación.

Hemos podido determinar que el estudio personal permite al estudiante compensar algunas deficiencias detectadas en el aprendizaje rápido, significando un indudable factor de mejora global del aprendizaje. En la adquisición rápida de conocimientos se ha demostrado más efectiva la explicación oral sin iconografía que con ella y más asequibles las preguntas conceptuales que las que piden nombrar o identificar a partir de unas imágenes ${ }^{1}$. El estudio personal ha permitido obtener mejoras en las notas de estos tipos de cuestiones de modo que pueden ser consideradas estadísticamente iguales (Tabla 1)

Las cuestiones que requirieron una actitud activa por parte de los alumnos durante la actividad docente, han obtenido un grado de mejoría significativamente mayor que aquellas que sólo precisaban una actitud de observación (Tabla 1) En la mayoría de esas cuestiones los alumnos tomaron sus apuntes que después utilizaron para preparar el examen, demostrándose que estos documentos pueden ser útiles para su estudio diferido ${ }^{4}$.

La comunicación no verbal del profesor o "lenguaje del cuerpo" ha sido considerada metafóricamente "el medio ambiente natural de las palabras". Tiene repercusión, no tan sólo para el alumno, sino también en la calidad de la expresión verbal del orador $^{6}$. Nuestros resultados parecen mostrar que no sólo es un elemento didáctico favorable en el aprendizaje rápido $^{1}$ sino que su influencia persiste en el tiempo. Así hemos observado que el binomio percepción auditiva más actitud de observación, ha mostrado ser el más útil para mejorar los resultados tanto de las cuestiones enseñadas sin soporte iconográfico como los de aquellas en que el alumno no debía tomar apuntes ni efectuar ejercicio alguno de secuenciación (Tabla 1) En estos casos los alumnos pudieron centrar su atención en el profesor, eliminando posibles factores de distracción, como el atribuido al uso de las imágenes visuales ${ }^{7}$.

La comunicación no verbal del profesor se ejerce puntualmente durante la explicación de un determinado tema y no queda recogida en los apuntes, ni en la grabadora de voz de los estudiantes y a pesar de esto sigue mostrándose un factor contingente para el aprendizaje, de lo cual cabe deducir que su impronta en el cerebro de los alumnos sufre una "dilución" temporal lenta. En nuestra opinión está expresividad del profesor sería uno de los factores más relevantes que justificaría por qué la actividad docente en el aula influencia y media en el autoaprendizaje posterior del alumno ${ }^{2}$.

En el ámbito lingüístico hemos observado que la complejidad de un término representa una mayor desventaja para el aprendizaje que la dificultad fonética (Tabla 1) Después del estudio personal ha seguido siendo más difícil aprender los términos complejos que los simples, pero en cambio se ha conseguido una 
Tabla 2. Resumen de los resultados estadísticos según el tiempo transcurrido entre ambas pruebas

\begin{tabular}{|c|c|c|c|}
\hline $\begin{array}{l}\text { Número de alumnos según los días de que } \\
\text { dispusieron para el estudio personal }\end{array}$ & $\mathrm{N}$ & Mediaa & Desviación típica \\
\hline$<=16$ días & 45 & 3.8 & 2.1 \\
\hline$>16$ días & 21 & 3.3 & 2.1 n.s. \\
\hline
\end{tabular}

${ }^{a}$ Diferencias de puntuación global obtenida entre ambas pruebas en escala de $0-10 .{ }^{*} p<0.05 . n s$, no diferencias significativas

mejora significativamente mayor en el aprendizaje de términos con dificultad fonética alta que la obtenida en los de dificultad menor (Tabla 1). Toda la información comunicada por medio de lenguaje oral o escrito es codificada y almacenada en forma de proposiciones verbales (memoria semántica) y requiere de la abstracción de conceptos, reglas y principios aunque haya ido acompañada de imágenes o de vinculaciones previas particulares ${ }^{8}$. La dificultad específica de los términos complejos puede radicar en que el alumno ha de aprender dos prefijos, conceptualmente independientes, e integrarlos en una nueva palabra que le debe evocar un nuevo concepto (p. ej. los prefijos -Toraco- onfalo en toracoonfalópago)

En este estudio hemos valorado cómo influye el tiempo transcurrido entre la sesión de la actividad docente y evaluación diferida, no habiendo diferencias significativas en la mejora de la nota global en función de la separación temporal (Tabla 2) De este modo el papel positivo del estudio personal ha resultado homogeneizador frente al papel diluyente en la memoria que se podría atribuir al paso del tiempo o a la interferencia ejercida por los nuevos aprendizajes ${ }^{8}$.

Como conclusión podemos decir que el diseño de nuestro trabajo, comparando los resultados obtenidos tras contestar las mismas preguntas antes y después de disponer de tiempo para el estudio personal, nos ha permitido evidenciar el importante papel que tiene este factor en la consolidación de la comprensión de las diversas cuestiones, con independencia del grado de dificultad que representó su aprendizaje rápido.

\section{BIBLIOGRAFIA}

1. Nebot-Cegarra J, Campillo M, Pérez J. Influencia de la metodología docente en la adquisición rápida de conocimientos. Educación Médica 2003; 6:31-37.

2. Wood D, Underwood J, Avis P. Integrated learning systems in the classroom. Comput Educ 1999; 33:91-108.

3. Brett P. A comparative study of the effects of the use of multimedia on listening comprension. System 1997; 25:39-53.

4. Castelló M, Monereo C. Com prenen apunts els estudiants?. Docència de Qualitat (Estudis i recerques) UAB 1999; ficha $\mathrm{n}^{\circ} 4$.

5. Birdwhistell RL. El lenguaje del cuerpo: el medioambiente natural de las palabras. En: Silverstein A, editor. Comunicación humana. Exploraciones teóricas. México DF: Trillas Editorial, 1985; 215-232.

6. Mc Clave EZ. Linguistic functions of head movements in the context of speech. J Pragmat 2000; 32:855-878.

7. Coniam D. The use of audio or video comprehension as an assessment instrument in the certification of English language teachers: a case study. System 2001; 29:1-14.

8. Good Th., Brophy P. Psicologia educativa contemporánea. México DF: Mc Graw-Hill Interamericana Editores, 1996. 